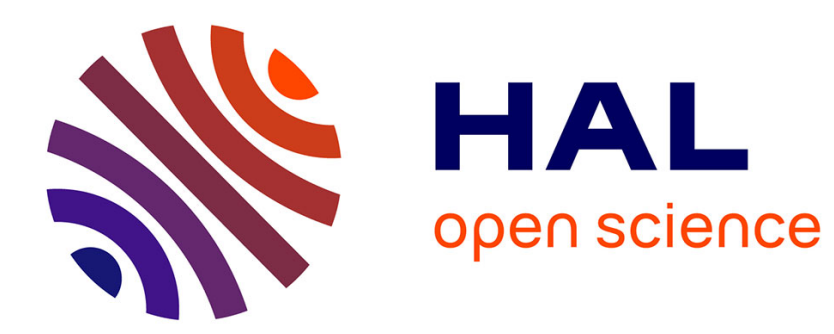

\title{
Convolution Surfaces based on Polygons for Infinite and Compact Support Kernels
}

\author{
Evelyne Hubert
}

\section{To cite this version:}

Evelyne Hubert. Convolution Surfaces based on Polygons for Infinite and Compact Support Kernels. Graphical Models, 2012, 74 (1), pp.1-13. 10.1016/j.gmod.2011.07.001 . inria-00536840

\section{HAL Id: inria-00536840 \\ https://hal.inria.fr/inria-00536840}

Submitted on 17 Nov 2010

HAL is a multi-disciplinary open access archive for the deposit and dissemination of scientific research documents, whether they are published or not. The documents may come from teaching and research institutions in France or abroad, or from public or private research centers.
L'archive ouverte pluridisciplinaire HAL, est destinée au dépôt et à la diffusion de documents scientifiques de niveau recherche, publiés ou non, émanant des établissements d'enseignement et de recherche français ou étrangers, des laboratoires publics ou privés. 


\title{
Convolution Surfaces based on Polygons for Infinite and Compact Support Kernels
}

\author{
Evelyne Hubert \\ INRIA Méditérranée - GALAAD \\ http://www.inria.fr/members/Evelyne.Hubert
}

November 17, 2010

\begin{abstract}
We provide mathematical formulae to create 3D smooth shapes fleshing out a skeleton made of line segments and polygons. The boundary of the shape created is a level set of a convolution function. This latter is obtained through the integration of a kernel function along the skeleton. The technique has been proposed and developed for geometric modeling in computer graphics.

Given the additivity of integration, the convolution function of a complex skeleton is the sum of the convolution functions for its simpler elements. Providing the closed form formulae for the convolution function generated by a polygon is the main contribution of the present paper. We apply Green's theorem and improve on previous results in several ways. First we do not require the prior triangulation of the polygon. Then, we obtain formulae for complete families of kernels, either with infinite or compact supports. The families are indexed by an integer that controls sharpness and smoothness. Full generality is achieved through recurrence formulae. Last, but not least, the geometric computations needed, in the case of compact support kernels, are restricted to intersections of spheres with line segments, rather than intersections of spheres with triangles in previous works.
\end{abstract}

keyword Green's theorem; Polygonal Mesh; Implicit Surfaces; Convolution Surfaces; Geometric Modelling; Computer Graphics; Recurrences; Symbolic Integration. 


\section{Introduction}

Convolution is a technique used in Computer Graphics to generate smooth 3D volumes around a skeleton of lower dimension $[2,3,4,6,7,22,10,13]$. Convolution surfaces are defined as level sets of a convolution function obtained by integrating a kernel function along this skeleton. Given the additivity of integration, the convolution function of a complex skeleton is the sum of the convolution functions for its simpler elements. One-dimensional skeletons create tubular like volume. Being able to handle additionally two-dimensional skeletons allows more generality on the shape to be generated [24]. To allow interactive modeling, the technique has relied on closed form formulae for the convolution functions generated by basic skeleton elements such as points, line segments, arcs of circle and triangles. Those formulae were often obtain through symbolic integration, as implemented by computer algebra software [9, 5], on a case by case basis. Several alternative to the Gaussian [3, 4] were proposed as kernel functions in order to obtain those. They can be split into kernels with infinite support $[7,12,15,26]$ or compact support $[18,27]$. In this paper we provide general formulae for convolution functions generated by a polygon with families of both types of kernels.

We consider families of kernels indexed by an integer. The families of Cauchy and power inverse kernels can actually be treated uniformly, as was shown in [11] for line segments. The index controls the sharpness. We introduce in this paper a family of compact support kernels, which generalizes the kernel function used in $[23,13]$. The index then controls the smoothness.

We reduce the integration along a planar region to a contour integral thanks to Green's theorem. That allows us to improve on previous results converning convolution of polygons in several ways. First, we obtain formulae for complete families of kernels, either with infinite or compact supports. Second, we do not require the prior triangulation of the polygon. Last, but not least, the geometric computations needed, in the case of compact support kernels, are restricted to the intersection of spheres with line segments, rather than intersection of spheres with triangles in previous works. We thus provide major improvements over both $[15,13]$. As argued in this latter article, we can envision the application of the present results in restoring regular implicit surfaces from polygon soups [20, 14].

In next section we introduce the families of kernels we shall consider and we define mathematically the notion of a convolution function based on a one or two dimensional skeleton regular patch. In Section 3 we review the general results obtained for line segments in [11] and extend those to the family of compact support kernels considered. The case of line segments allows us to illustrate in Section 4 the point of having different types of kernels and families of those. In Section 5 we introduce the strategy we use to obtain the convolution functions for planar polygon. This strategy is carried on the family of power inverse kernels in Section 6 and the family of compact support kernels in Section 8. The case of Cauchy kernels is identical to the case of power inverse kernels and we comment on this in Section 7 .

\section{Convolution surfaces}

A convolution surface in $\mathbb{R}^{3}$ is the level set of a function that results from integrating a kernel function $K$ along a skeleton $\mathcal{S}$, which is a set of lower dimensional geometric elements: points, bounded curves and surfaces. The additivity property of integration allows superposition: we can concentrate on obtaining the convolution function for each type of skeleton elements. The convolution function for the whole skeleton is obtained by summing the convolution functions obtained for each of its elements.

In this section we introduce families of kernels members of which arose in computer graphics litterature and are of practical use. We then define precisely the convolution functions generated by one and two dimensional regular skeleton patches. 


\section{$2.1 \quad$ Kernels}

The kernels in use in the literature are given by functions $K: \mathbb{R}^{+} \rightarrow \mathbb{R}^{+}$that are at least continously differentiable. The argument is the distance between a point in space and a point on the skeleton. Those kernels are decreasing functions on $\mathbb{R}^{+}$and strictly decreasing when non zero: $K(r)>0 \Rightarrow K^{\prime}(r)<0$.

The first convolution surfaces that appeared in computer graphics $[3,4]$ were based on the Gaussian kernel: $r \mapsto e^{-s r^{2}}$ that depends on a parameter $s>0$. The difficulty in evaluating the resulting convolutions prompted the introduction of kernels that provided closed form convolution functions on basic skeletal elements. [15, 23] promoted the Cauchy kernel $r \mapsto \frac{1}{\left(1+s r^{2}\right)^{2}}$ after [26] introduced the inverse function $r \mapsto \frac{1}{r}$. For faster convolution [7, 10] introduced the inverse cube kernel $r \mapsto \frac{1}{r^{3}}$. [12] also exhibited the benefit of using the quintic inverse $r \mapsto \frac{1}{r^{5}}$.

In this paper we study uniformly the convolution with Cauchy type and power inverse kernels which are the families indexed by $i \in \mathbb{N} \backslash\{0\}$ given by

$$
\mathfrak{c}_{s}^{i}: r \mapsto \frac{1}{\left(1+s r^{2}\right)^{\frac{i}{2}}} \quad \text { and } \quad \mathfrak{p}^{i}: r \mapsto \frac{1}{r^{i}} .
$$

From an algebraic point of view there is little difference on the convolution function obtained. This can be seen from a qualitative persective by introducing the family of kernels

$$
\mathfrak{p}_{s}^{i}: r \mapsto \frac{1}{\left(\frac{1}{s}+r^{2}\right)^{\frac{i}{2}}}
$$

for which $\mathfrak{p}^{i}$ is a limiting case, as $s$ tends to $\infty$. We have $\mathfrak{p}_{s}^{i}=s^{\frac{i}{2}} \mathfrak{c}_{s}^{i}$. When $r=0, \mathfrak{c}_{s}^{i}$ is always equal to 1 and $\mathfrak{p}_{s}^{i}$ is equal to $s^{\frac{i}{2}}$ while $\mathfrak{p}^{i}=\mathfrak{p}_{0}^{i}$ is infinite.

Those kernels decrease relatively fast with distance but have an infinite support and can lead to unwanted bulges or blending. Kernels with compact support allow to avoid those as a given point on the skeleton has a finite radius $R$ of influence.

Several piecewise polynomial functions were used in the litterature $[18,27,23]$. Their use necessitates to determine the geometry of the intersection of the skeleton with spheres. This is amenable for skeletons made of line segments or even arcs of circle. This is already more challenging in the presence of triangles in the skeleton. In $[23,13]$ the different configurations of the intersection of a triangle with a sphere are considered. Our strategy considerably simplifies the geometric computations as only the boundary of the polygon needs to be intersected with the spheres.

We consider the family of compact support kernels

$$
\mathfrak{k}_{R}^{i}: r \mapsto\left\{\begin{array}{ll}
\left(1-\frac{r^{2}}{R^{2}}\right)^{\frac{i}{2}} & \text { if } r<R \\
0 & \text { otherwise }
\end{array} .\right.
$$

To obtain convolution surfaces that are at least continuously differentiable we consider only those kernels for $i \geq 3$. For $i<3, \mathfrak{k}_{R}^{i}$ is not differentiable at $r=R$. The case $i=4$ is actually the case considered in [23] and [13], with the claim that it is commonly used for modeling blobby objects in public domain ray-tracing programs (see [23, Section 2.7]). As we increase $i$ though, we obtain smoother shapes.

\subsection{Regular skeleton patches}

We assume that the skeleton can be partitioned into regular patches defined by regular curves or surfaces in $\mathbb{R}^{3}$ parameterized by connected compact sets of $\mathbb{R}$ or $\mathbb{R}^{2}$ respectively. The convolution function for the skeleton is then obtained by summing the convolution functions for each of those patches. 

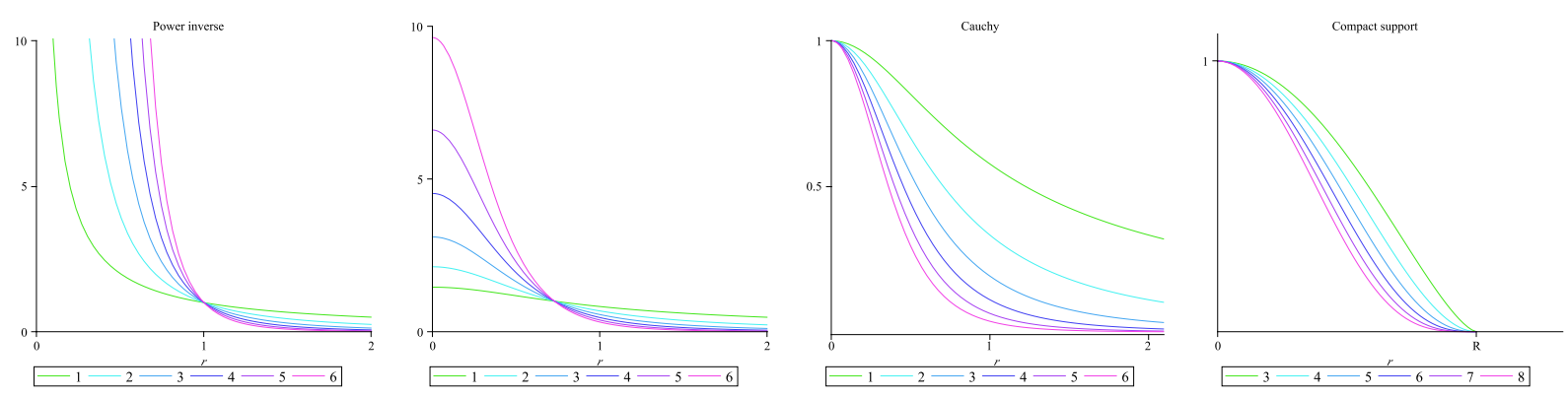

Figure 1: The graphs of the kernel functions $\mathfrak{p}^{i}, \mathfrak{p}_{s}^{i}, \mathfrak{c}_{s}^{i}$ and $\mathfrak{k}_{R}^{i}$, varying $i$.

Consider first a parameterized regular curve $\Gamma:[a, b] \subset \mathbb{R} \rightarrow \mathbb{R}^{3}$. It is a regular curve if $\Gamma$ is continuously differentiable and $\Gamma^{\prime}$ doesn't vanish: the infinitesimal arclength is $\left|\Gamma^{\prime}(t)\right| \mathrm{d} t$. The convolution function based on $\mathcal{S}=\Gamma([a, b])$ at a point $P \in \mathbb{R}^{3}$ is then defined by

$$
\mathcal{C}_{\Gamma}^{K}(P)=\int_{a}^{b} K(|P \Gamma(t)|)\left|\Gamma^{\prime}(t)\right| \mathrm{d} t .
$$

The integral is independent of the (regular) parametrisation of the curve used. For a uniform notation we note $\Omega=[a, b]$ when considering that type of skeleton patch.

Consider now a surface parameterized by $\Gamma: \Omega \subset \mathbb{R}^{2} \rightarrow \mathbb{R}^{n}$, where $\Omega$ is a connected compact set. The infinitesimal area is given by $[1$, Section 6.4$]$ :

$$
\left|\Gamma_{u} \times \Gamma_{v}\right| \mathrm{d} u \mathrm{~d} v=\sqrt{\left|\begin{array}{ll}
\Gamma_{u} \cdot \Gamma_{u} & \Gamma_{u} \cdot \Gamma_{v} \\
\Gamma_{u} \cdot \Gamma_{v} & \Gamma_{v} \cdot \Gamma_{v}
\end{array}\right|} \mathrm{d} u \mathrm{~d} v .
$$

The parametrisation is regular if this quantity does not vanish in $\Omega$. Then the convolution function based on $\mathcal{S}=\Gamma(\Omega)$ at a point $P \in \mathbb{R}^{3}$ is defined by

$$
\mathcal{C}_{\Gamma}^{K}(P)=\iint_{\Omega} K(|P \Gamma(u, v)|)\left|\Gamma_{u} \times \Gamma_{v}\right| \mathrm{d} u \mathrm{~d} v .
$$

Proposition 2.1 Consider a skeleton regular patch parameterized by $\Gamma: \Omega \rightarrow \mathbb{R}^{3}$. If for all $q \in \Omega$, $P \mapsto K(|P \Gamma(q)|)$ is $k$-time continuously differentiable in a domain $\left[a_{1}, b_{1}\right] \times\left[a_{2}, b_{2}\right] \times\left[a_{3}, b_{3}\right]$ of $\mathbb{R}^{3}$ then $\mathcal{C}_{\Gamma}^{K}$ is $k$-time continuously differentiable on the interior of this domain.

This comes from the Leibniz integral rule (see for instance [8], [17]) and actually entails that we can commute derivation and the integral sign.

Convolution functions obtained from a Cauchy kernel $\mathfrak{c}_{s}^{i}$ on a regular patch are therefore infinitely differentiable, while those obtained from a power inverse kernel $\mathfrak{p}^{i}$ are infinitely differentiable outside of the patch $\Gamma(\Omega)$. On the other hand the convolution function obtained with the compact support kernel $\mathfrak{k}_{R}^{i}$ is $\left\lfloor\frac{i}{2}\right\rfloor-1$ continuously differentiable only.

As the reverse image of a closed set by a $k$-continuously differentiable map, $k \geq 1$, the resulting convolution hyper-surfaces $\mathcal{C}_{\Gamma}^{K}(P)=\kappa$ are closed (in a topological sense) and smooth, provided $\kappa$ is not a critical value of $\mathcal{C}_{\Gamma}^{K}$. It is the boundary of a smooth 3-dimensional manifold $V_{c}$ defined by $\mathcal{C}_{\Gamma}^{K}(P) \geq \kappa$. Furthermore $V_{\kappa}$ and $V_{\kappa^{\prime}}$ are diffeomorphic provided that there is no critical values in the interval $\left[\kappa, \kappa^{\prime}\right]$ [16, Theorem 3.1]. 


\section{Convolution of line segments}

The geometrical properties of the convolution surfaces based on line segments were studied in [11]. General formulae for the convolution functions for the Cauchy and power inverse kernels were given there in a uniform manner. We briefly recall those results and extend them to the family of compact support kernels considered.

Let us mention that for any kernel function $K$ the point of the convolution surface $\mathcal{C}_{[A B]}^{K}(P)=c$ that is at a maximal distance from the line segment lies on the perpendicular bisector [11, Proposition 4.1]. This result can be used to adjust the level set to obtain a given thickness around the segment.

For power inverse and Cauchy kernels, this is a strict maximum. This can create some unwanted bulges and even blendings, as illustrated in Figure 5. For compact support kernels this maximum is flat for long enough line segments. One of the difficulty though with compact support kernels defined by piecewise polynomial functions is that for each point in space we need to determine the part of the skeleton that has a contribution to the convolution function at that point. In the case of skeletons made of line segments, we need to solve degree two equations. Yet, small changes in the skeleton then only have local impact. This can be taken advantage of in animation.

We can evaluate the convolution function based on a line segment with any power inverse, Cauchy or compact support kernels through a single recurrence.

\subsection{The fundamental integral and its recurrence formula}

In [11] it was shown that the convolution function based on a line segment with either the Cauchy or the power inverse kernel boils down to evaluating the same integral:

$$
\mathfrak{I}_{i}(a, b, c)=\int_{0}^{1} \frac{1}{\left(a t^{2}-2 b t+c\right)^{\frac{i}{2}}} \mathrm{~d} t
$$

with slightly different values for $a, b$ and $c$. A good level of generality for its evaluation is obtained by using the recurrence formula bearing on this integral [19]:

$$
(i-2)\left(c a-b^{2}\right) \mathfrak{I}_{i}(a, b, c)+a(3-i) \mathfrak{I}_{i-2}(a, b, c)=\frac{(a-b)}{(a-2 b+c)^{\frac{i-2}{2}}}+\frac{b}{c^{\frac{i-2}{2}}} .
$$

We can observe that the recurrence is still valid for $i \leq 0$. This entails that the convolution functions generated by a line segment with the family of compact support kernels $\mathfrak{k}_{R}^{i}$ can also be evaluated thanks to this recurrence. There is nonetheless a difference in the initialisation stage.

First, for the integral to be well defined we need to have $(a, b, c)$ satify:

$$
\forall t \in[0,1], a t^{2}-2 b t+c>0 \text { when } i>0
$$

and

$$
\forall t \in[0,1], a t^{2}-2 b t+c \geq 0 \text { when } i \leq 0
$$

We distinguish the useful cases according to the discriminant. For the compact support kernel $\mathfrak{k}_{R}^{i}$ we are in the case where $b^{2}-a c>0$ while $a<0$. Thus $a t^{2}-2 b t+c \geq 0, \forall t \in[0,1]$, iff $-\sqrt{b^{2}-a c} \leq b \leq a+\sqrt{b^{2}-a c}$. Then ${ }^{1}$

$$
\mathfrak{I}_{1}(a, b, c)=\frac{-1}{\sqrt{-a}}\left(\arctan \left(\frac{a-b}{\sqrt{-a} \sqrt{a-2 b+c}}\right)+\arctan \left(\frac{b}{\sqrt{-a} \sqrt{c}}\right)\right) .
$$

\footnotetext{
${ }^{1}$ The formula in terms of arcsin analogous to the next one suffers a lack of differentiability.
} 
In the case of Cauchy kernels we have $b^{2}-a c<0$ and $a>0$. Then $a t^{2}-2 b t+c>0$ for all $t \in \mathbb{R}$ and

$$
\mathfrak{I}_{1}(a, b, c)=\frac{1}{\sqrt{a}}\left(\operatorname{arcsh}\left(\frac{a-b}{\sqrt{a c-b^{2}}}\right)+\operatorname{arcsh}\left(\frac{b}{\sqrt{a c-b^{2}}}\right)\right) .
$$

For power inverse kernels $a>0$ but $b^{2}-a c \leq 0$. The case $b^{2}-a c=0$ has a geometric meaning and is treated separately. For $\frac{b}{a} \notin[0,1]$

$$
\mathfrak{I}_{i}\left(a, b, \frac{b^{2}}{a}\right)= \begin{cases}\frac{1}{\sqrt{a}}\left|\ln \left(1-\frac{b}{a}\right)\right| & \text { for } i=1 \\ \frac{a^{\frac{i-2}{2}}}{1-i}\left(\frac{a-b}{|a-b|^{i}}+\frac{b}{|b|^{i}}\right) & \text { otherwise. }\end{cases}
$$

Note that from the recurrence equation we actually immediately see two simple cases. Substituting $i=2$ we verify that $\mathfrak{I}_{0}(a, b, c)=1$ while substituting $i=3$ we obtain:

$$
\mathfrak{I}_{3}(a, b, c)=\frac{1}{c a-b^{2}}\left(\frac{a-b}{\sqrt{a-2 b+c}}+\frac{b}{\sqrt{c}}\right) .
$$

It follows that all the $\mathfrak{I}_{2 i+1}$, for $i \geq 1$, involve only radical functions on top of arithmetic operations while $\mathfrak{I}_{-2 i}$, for $i \leq 0$, are rational functions.

The only additional piece of information we need to cover all the cases arising in the convolution of line segments is:

$$
\mathfrak{I}_{2}(a, b, c)=\frac{1}{\sqrt{a c-b^{2}}}\left(\arctan \left(\frac{a-b}{\sqrt{a c-b^{2}}}\right)+\arctan \left(\frac{b}{\sqrt{a c-b^{2}}}\right)\right) \text { when } b^{2}-a c<0 .
$$

\subsection{Power inverse kernels}

Two points $A, B \in \mathbb{R}^{3}$ define the line segment $[A B]$. A regular parametrisation for this line segment is given by $\Gamma:[0,1] \rightarrow \mathbb{R}^{3}$ with $\Gamma(t)=A+(B-A) t$. Therefore for a point $P \in \mathbb{R}^{3}$ we have

$$
|P \Gamma(t)|^{2}=|A B|^{2} t^{2}-2 \overrightarrow{A B} \cdot \overrightarrow{A P} t+|A P|^{2}
$$

The convolution function at a point $P \in \mathbb{R}^{3}$ based on $[A B]$ with the power inverse kernel $\mathfrak{p}^{i}$ is thus given by

$$
\mathcal{C}_{[A B]}^{\mathfrak{p}^{i}}(P)=|A B| \mathfrak{I}_{i}\left(|A B|^{2}, \overrightarrow{A B} \cdot \overrightarrow{A P},|A P|^{2}\right) .
$$

It is actually be practical to introduce the functions $\mathcal{D}_{[A B]}^{\mathfrak{p}^{i}}(P)=\mathfrak{I}_{i}\left(|A B|^{2}, \overrightarrow{A B} \cdot \overrightarrow{A P},|A P|^{2}\right)$ as they naturally appear in the convolution of polygons as well.

For a geometric understanding of the quantities apearing in the evaluation of $\mathcal{C}_{[A B]}^{\mathfrak{p}^{i}}(P)$, it is worth mentioning that, if $a=|A B|^{2}, b=\overrightarrow{A B} \cdot \overrightarrow{A P}$ and $c=|A P|^{2}$ then $a-2 b+c=|B P|^{2}, a-b=\overrightarrow{B A} \cdot \overrightarrow{B P}$, and $c a-b^{2}=|A B|^{2}|H P|^{2}$, where $H$ is the projection of $P$ on the line $(A B)$. The case $b^{2}-a c=0$ thus corresponds to $P \in(A B)$. When furthermore $P \in[A B]$ we have $\frac{b}{a} \in[0,1]$. It follows that, for $i>1$, $\mathcal{C}_{[A B]}^{\mathfrak{p}^{i}}(P)$ tends to infinity as $P$ approaches $[A B]$. That ensures that the line segment $[A B]$ is always included in the bounded volume $\mathcal{C}_{[A B]}^{\mathfrak{p}^{i}}(P) \geq \kappa>0$.

According to [11, Proposition 4.1] if we want to obtain a given thickness $\tau$ for a segment of length $l$ we need to take $\kappa=l \mathfrak{I}_{i}\left(l^{2}, \frac{l^{2}}{2}, \frac{l^{2}}{4}+\tau^{2}\right)$. 


\subsection{Cauchy kernels}

It was shown in [11] that the algebraic treatment for the Cauchy kernel is exactly the same as for power inverse kernels. The convolution function at a point $P \in \mathbb{R}^{3}$ based on the line segment $[A B]$ with the Cauchy kernel $\mathfrak{c}_{s}^{i}$ is given by the integral of Section 3.1:

$$
\mathcal{C}_{[A B]}^{\mathfrak{c}_{s}^{i}}(P)=|A B| \mathfrak{I}_{i}\left(s|A B|^{2}, s \overrightarrow{A B} \cdot \overrightarrow{A P}, 1+s|A P|^{2}\right) .
$$

Here, the function $\mathcal{C}_{[A B]}^{\mathfrak{c}_{s}^{i}}$ is well defined for all $P \in \mathbb{R}^{3}$. Yet it is a bounded function. Its maximum is reached at the mid point of $[A B]$. That entails that the level sets of the convolution function need not include the whole line segment.

For a segment of length $l$, if we want to obtain a given thickness $\tau$ we need to take the level set $\kappa=$ $l \Im_{i}\left(s l^{2}, s \frac{l^{2}}{2}, 1+s\left(\frac{l^{2}}{4}+\tau^{2}\right)\right)$ [11, Proposition 4.1]. Yet, if $l \mathfrak{I}_{i}\left(s l^{2}, 0,1\right) \leq \kappa \leq l \mathfrak{I}_{i}\left(s l^{2}, s \frac{l^{2}}{2}, 1+s\left(\frac{l^{2}}{4}\right)\right)$, the convolution surface will intersect the line segment $[A B]$. As for $\kappa>l \mathfrak{I}_{i}\left(s l^{2}, s \frac{l^{2}}{2}, 1+s\left(\frac{l^{2}}{4}\right)\right)$ the convolution surfaces is empty.

As before we introduce here the functions that arise in the convolution of polygons, namely:

$$
\mathcal{D}_{[A B]}^{\mathfrak{c}_{s}^{i}}(P)=\frac{1}{|A B|} \mathcal{C}_{[A B]}^{\mathfrak{c}_{s}^{i}}(P)=\mathfrak{I}_{i}\left(s|A B|^{2}, s \overrightarrow{A B} \cdot \overrightarrow{A P}, 1+s|A P|^{2}\right)
$$
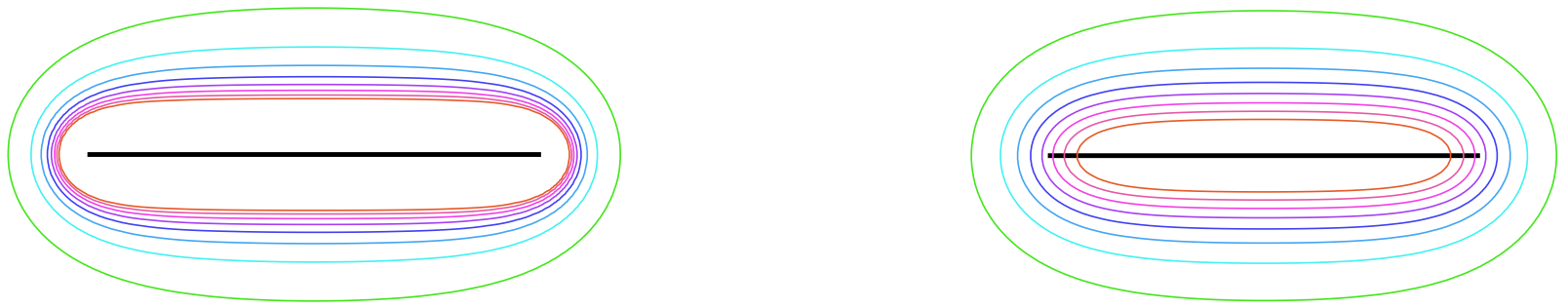

Figure 2: Different level sets of the convolution function for a line segment with a cubic inverse power (left) and the classical Cauchy kernel (right).

\subsection{Compact support kernels}

In the case of the compact support kernels $\mathfrak{k}_{R}^{i}$, with $i$ even, there is no difficulty in integration since we deal with polynomials. To compute $\mathcal{C}_{[A B]}^{\mathfrak{k}_{R}^{i}}$ at a point $P \in \mathbb{R}^{3}$ we nonetheless need to determine $A^{P}$ and $B^{P}$ that are the end points of the intersection of the sphere centered at $P$ of radius $R$ with the line segment $[A B]$. We can take the convention $A_{P}=B_{P}$ when this intersection is empty. Then:

$$
\mathcal{C}_{[A B]}^{\mathfrak{k}_{R}^{i}}(P)=\left|A_{P} B_{P}\right| \int_{0}^{1}\left(1-\frac{|P \Gamma(t)|^{2}}{R^{2}}\right)^{\frac{i}{2}} \mathrm{~d} t
$$

where $\Gamma(t)=A_{P}+\left(B_{P}-A_{P}\right) t$.

Thus

$$
\mathcal{C}_{[A B]}^{\mathfrak{k}_{R}^{i}}(P)=\left|A_{P} B_{P}\right| \mathfrak{I}_{-i}\left(-\frac{\left|A_{P} B_{P}\right|^{2}}{R^{2}},-\frac{\overrightarrow{A_{P} P} \cdot \overrightarrow{A_{P} B_{P}}}{R^{2}}, 1-\frac{\left|A_{P} P\right|^{2}}{R^{2}}\right)
$$


As previously we shall introduce here the functions that arise in the convolution of polygons:

$$
\mathcal{D}_{[A B]}^{\mathfrak{k}_{R}^{i}}(P)=\left\{\begin{array}{l}
0 \text { when } A_{P}=B_{P} \\
\frac{\mathcal{C}_{[A B]}^{\mathfrak{k}_{R}^{i}}(P)}{\left|A_{P} B_{P}\right|}=\mathfrak{I}_{-i}\left(-\frac{\left|A_{P} B_{P}\right|^{2}}{R^{2}},-\frac{\overrightarrow{A_{P} P} \cdot \overrightarrow{A_{P} B_{P}}}{R^{2}}, 1-\frac{\left|A_{P} P\right|^{2}}{R^{2}}\right) \text { otherwize }
\end{array}\right.
$$

For any line segment $[A B]$ it is impossible to achieve a thickness greater or equal to $R$. If the line segment has a length greater than $2 \sqrt{R^{2}-\tau^{2}}$, where $\tau<R$ is the desired thickness, then the convolution surface contains a cylindrical section around its middle part. Indeed, the length of the intersection of the line segment with the spheres centered at the points on the perpendicular bisector at distance $\tau$ remains constant as we move away from those parallely to the line segment. This is illustrated, and probaby clearer, in Figure 3 and compared to the results obtained with the power inverse kernels.
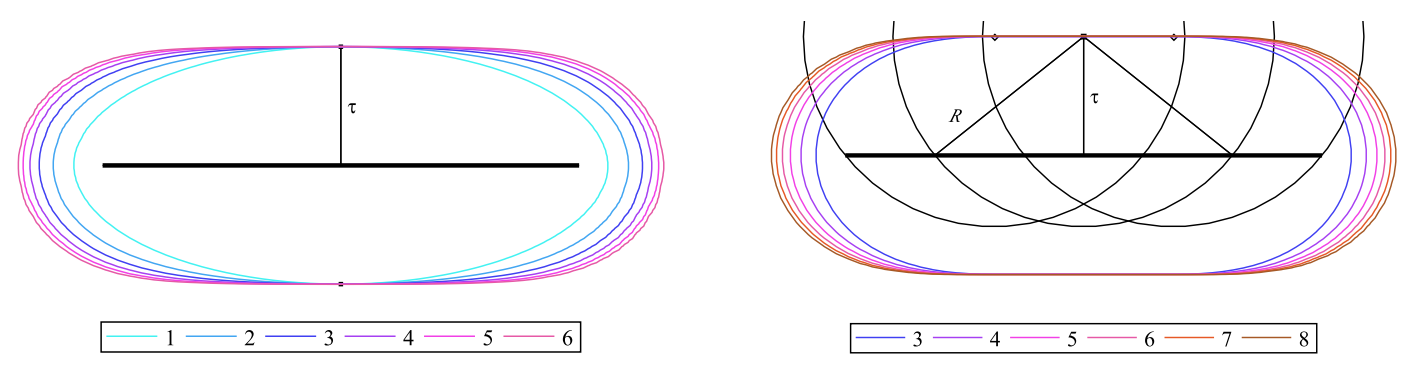

Figure 3: Convolution curves generated by a line segments of a prescribed thickness $\tau$ for power inverse (left) and compact support (right) kernels with varying $i$. The line segment between the two diamonds belongs to the convolution curves. We draw the outline of the relevant $\operatorname{arcs}$ of circle of radius $R$ centered at those points.

\section{Why do we consider several families of kernels}

For compact support kernels $\mathfrak{k}_{R}^{i}$ the convolution surface smoothness increases with $i$, as this can be observed in Figure 3. With infinite support kernels the convolution surface is smooth almost everywhere. And yet we can achieve sharpness. For the power inverse kernels $\mathfrak{p}^{i}$, the convolution surface is sharper in the angles of the skeleton as $i$ increases. This is illustrated in Figure 4.

When there is a critical value of level set, i.e. when the surface is no longer smooth, chances are that there is a change in the topology of the convolution surface [16]. This is illustrated in Figure 5 where there is a transition between bulging to blending as we lower the level set value.

What Figure 5 illustrates is that compact support kernels allow to eliminate the influence of skeleton elements that are at distance more than $R$. When the use of infinite support kernels can introduce unwanted bulges or even blending.

\section{Convolution based on planar polygons}

We consider a polygon $\mathfrak{Q}$ the (ordered) vertices of which are $Q^{0}, \ldots, Q^{q-1}$ ordered so that the boundary $\partial \mathfrak{Q}$ of $\mathfrak{Q}$ is a simple closed polygonal curve joining $Q^{0}, \ldots, Q^{q-1}$ and then $Q^{0}$. The points $Q^{0}, \ldots, Q^{q-1}$ are assumed to belong to a plane. 

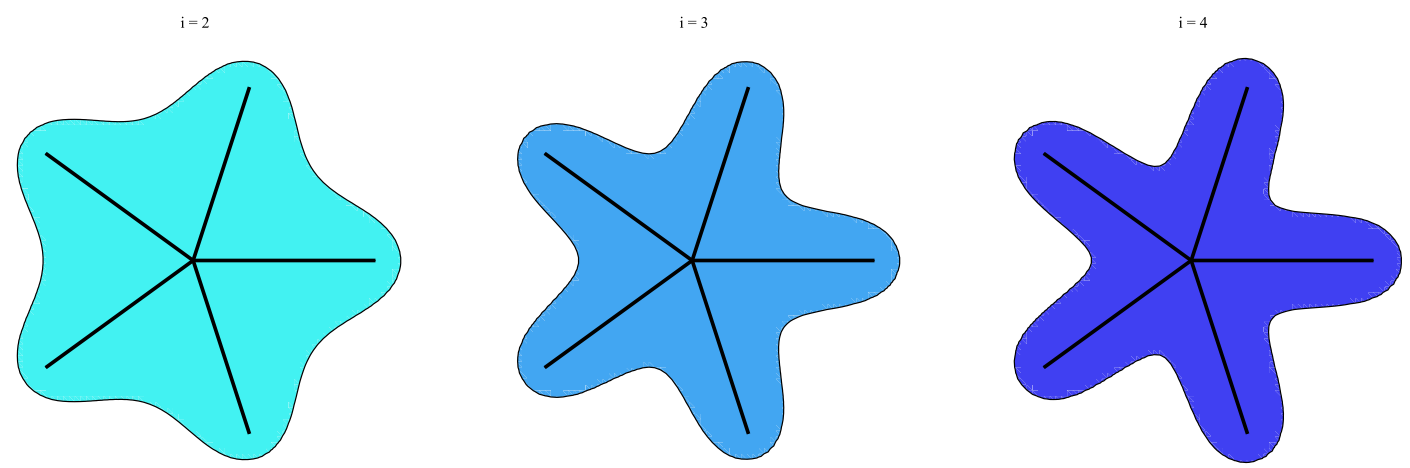

Figure 4: Convolution curves for a set of segments with power inverse kernel $\mathfrak{p}^{2}, \mathfrak{p}^{3}, \mathfrak{p}^{4}$. Sharpness increases from left to right.

We can define the unit normal to the polygon as:

$$
\overrightarrow{\mathbf{n}}=\frac{\overrightarrow{Q_{0} Q_{1}} \times \overrightarrow{Q_{0} Q_{2}}}{\left|\overrightarrow{Q_{0} Q_{1}} \times \overrightarrow{Q_{0} Q_{2}}\right|}=\frac{\overrightarrow{Q_{m} Q_{m+1}} \times \overrightarrow{Q_{m} Q_{m+2}}}{\left|\overrightarrow{Q_{m} Q_{m+1}} \times \overrightarrow{Q_{m} Q_{m+2}}\right|}
$$

where $\times$ is the cross-product in $\mathbb{R}^{3}$ and the indices are understood modulo $q$, that is $q \equiv 0, q+1 \equiv 1, \ldots$. Let $\bar{P}$ be the projection of $P$ on the plane of the polygon $\mathfrak{Q}$. The distance of $P$ to the plane of the polygon is

$$
|P \bar{P}|=\left|\overrightarrow{P Q_{0}} \cdot \overrightarrow{\mathbf{n}}\right|=\left|\overrightarrow{P Q_{m}} \cdot \overrightarrow{\mathbf{n}}\right| .
$$

We shall prove that for a kernel function $K$, the convolution function based on the polygon $\mathfrak{Q}=\left[Q^{0}, \ldots, Q^{q-1}\right]$ is given by

$$
\mathcal{C}_{\mathfrak{Q}}^{K}(P)=\sum_{m=0}^{q-1}\left(\overrightarrow{Q_{m} Q_{m+1}} \times \overrightarrow{Q_{m} P}\right) \cdot \overrightarrow{\mathbf{n}} \int_{0}^{1} G^{K}\left(Q_{m}-P+t\left(Q_{m+1}-Q_{m}\right)\right) \mathrm{d} t
$$

where $G^{K}: \mathbb{R}^{3} \rightarrow \mathbb{R}$ is a continuously differentiable function that is a solution of the partial differential equation

$$
u \frac{\partial G^{K}}{\partial u}(u, v, w)+v \frac{\partial G^{K}}{\partial v}(u, v, w)+2 G^{K}(u, v, w)=K\left(\sqrt{u^{2}+v^{2}+w^{2}}\right) .
$$

We first show how the convolution function of a planar skeleton patch can be reduced the integral along its one dimensional boundary. We then examine this integral along line segments. The result is then obtained by considering the boundary of the polygon on as a union of line segments.

In order to establish the formulae we first choose a positively oriented orthonormal frame $(\overrightarrow{\mathbf{u}}, \overrightarrow{\mathbf{v}}, \overrightarrow{\mathbf{n}})$ for $\mathbb{R}^{3}$ providing coordinates $(u, v, w)$ such that the concerned polygon $\mathfrak{Q}$ lies in the $(u, v)$ plane.

\subsection{Planar skeleton patches}

We consider here a 2-dimensional skeleton regular patch $\mathcal{S}$ given by a bounded region $\Omega$ in the plane $(O,(\overrightarrow{\mathbf{u}}, \overrightarrow{\mathbf{v}}))$. If $(x, y, z)$ are the coordinates of $P \in \mathbb{R}^{3}$ in the frame $(\overrightarrow{\mathbf{u}}, \overrightarrow{\mathbf{v}}, \overrightarrow{\mathbf{n}})$, the convolution function at $P$ is defined by

$$
\mathcal{C}_{\mathcal{S}}^{K}(P)=\iint_{\Omega} K\left(\sqrt{(u-x)^{2}+(v-y)^{2}+z^{2}}\right) \mathrm{d} u \mathrm{~d} v
$$



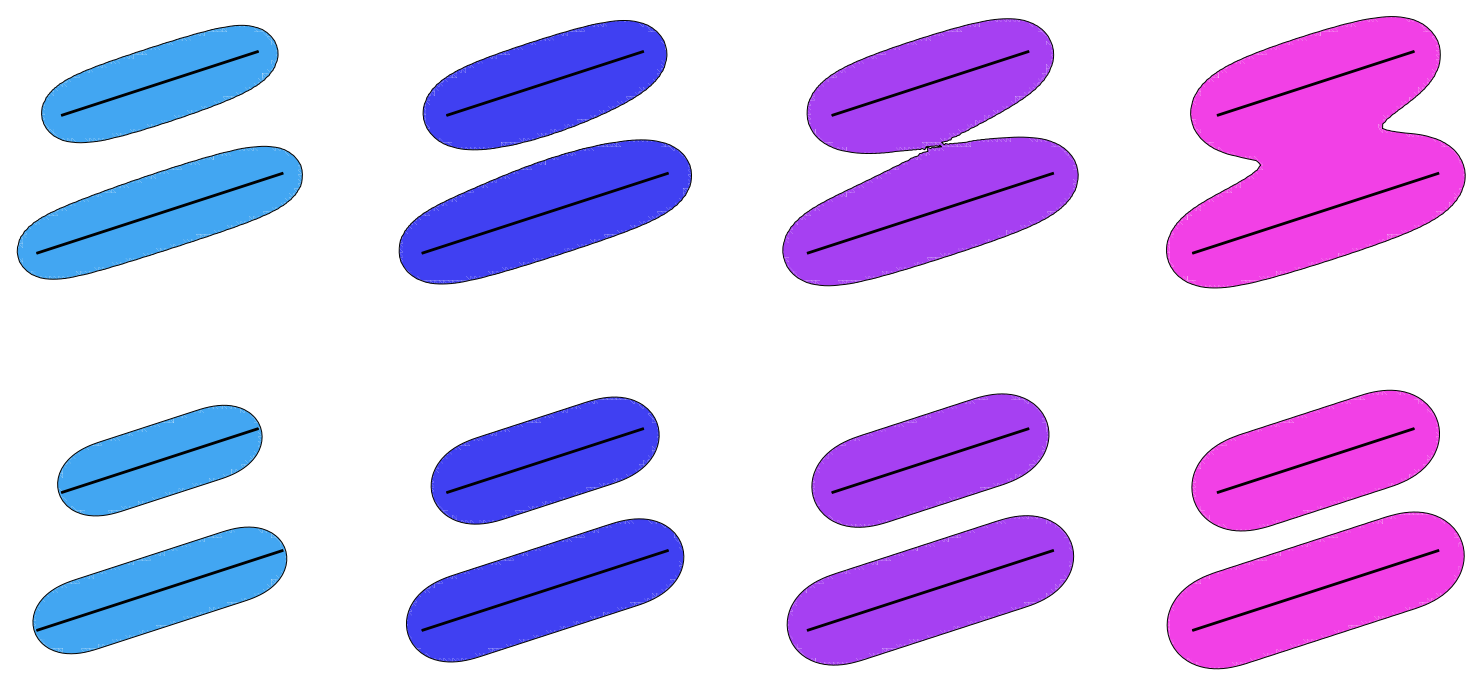

Figure 5: Convolution curves based on two parallel segments. The top line uses the cubic inverse kernel $\mathfrak{p}^{3}$ and the bottom line a compact support kernel $\mathfrak{k}_{R}^{3}$. Columns correspond to an identical sought thickness. For the cubic inverse kernel, a bulge and then a blend appear between the line segments, while the convolution surfaces get only thicker with the compact support kernel.

Green's theorem states that an integral over a bounded domain of $\mathbb{R}^{2}$ can be expressed as a contour integral along the boundary of this domain. More precisely let $\Omega$ be the region in $\mathbb{R}^{2}$ bounded by a piecewise smooth, simple closed curve $\partial \Omega$. Assume $f$ and $g$ are defined on an open region containing $\Omega$ and have continuous partial derivatives there. Then

$$
\oint_{\partial \Omega} f(u, v) \mathrm{d} u+g(u, v) \mathrm{d} v=\iint_{\Omega}\left(\frac{\partial g}{\partial u}-\frac{\partial f}{\partial v}\right) \mathrm{d} u \wedge \mathrm{d} v
$$

with $\partial \Omega$ positively oriented. We shall thus look for a continuously differentiable function $G^{K}: \mathbb{R}^{3} \backslash\{0\} \rightarrow \mathbb{R}$, such that

$$
u \frac{\partial G^{K}}{\partial u}(u, v, z)+v \frac{\partial G^{K}}{\partial v}(u, v, z)+2 G^{K}(u, v, z)=K\left(\sqrt{u^{2}+v^{2}+z^{2}}\right) .
$$

Then

$$
\mathcal{C}_{\mathcal{S}}^{K}(P)=\oint_{\partial \Omega} G^{K}(u-x, v-y, z)((u-x) \mathrm{d} u+(v-y) \mathrm{d} v) .
$$

Such a solution is provided by the homotopy operator [25, Section 5.2]:

$$
G^{K}(u, v, z)=\int_{0}^{1} t K\left(\sqrt{t^{2}\left(u^{2}+v^{2}\right)+z^{2}}\right) \mathrm{d} t .
$$

We shall provide an appropriate $G^{K}$ for the family of kernels with compact support and of power inverse kernels. The convolution functions for the Cauchy kernels are obtained likewise.

To obtain the convolution function $\mathcal{C}^{K}$ we additionally need to determine the restriction of the 1-form $((u-x) \mathrm{d} v-(v-y) \mathrm{d} u)$ on the contour $\partial \Omega$ defining the boundary of $\Omega$. In order to handle polygons, we treat the case of line segments. 


\subsection{Integral along a line segment}

Consider two points $A=\left(a_{1}, a_{2}, 0\right)$ and $B=\left(b_{1}, b_{2}, 0\right)$ in the $(u, v)$-plane. A parametrization for the segment $[A, B]$ is given by $\Gamma: t \mapsto A+(B-A) t$, so that $\Gamma^{*} u=a_{1}+\left(b_{1}-a_{1}\right) t$ and $\Gamma^{*} v=a_{2}+\left(b_{2}-a_{2}\right) t$. Therefore

$$
\Gamma^{*}((u-x) d v-(v-y) d u)=\left(\left(b_{1}-a_{1}\right)\left(y-a_{2}\right)-\left(b_{2}-a_{2}\right)\left(x-a_{1}\right)\right) \mathrm{d} t
$$

and we observe that

$$
\left(b_{1}-a_{1}\right)\left(x-a_{2}\right)-\left(b_{2}-a_{2}\right)\left(y-a_{1}\right)=\left|\begin{array}{ccc}
b_{1}-a_{1} & x-a_{1} & 0 \\
b_{2}-a_{2} & y-a_{2} & 0 \\
0 & z & 1
\end{array}\right|=\operatorname{det}(\overrightarrow{A B}, \overrightarrow{A P}, \overrightarrow{\mathbf{n}})=(\overrightarrow{A B} \times \overrightarrow{A P}) \cdot \overrightarrow{\mathbf{n}} .
$$

If $(x, y, z)$ are the coordinates of $P$ in the frame $(O, \overrightarrow{\mathbf{u}}, \overrightarrow{\mathbf{v}}, \overrightarrow{\mathbf{n}})$ then

$$
\begin{aligned}
\mathcal{C}_{\mathfrak{Q}}^{K}(P) & =\iint_{\mathfrak{Q}} K\left(\sqrt{(u-x)^{2}+(v-y)^{2}+z^{2}}\right) \mathrm{d} u \mathrm{~d} v \\
& =\sum_{m=0}^{q-1} \int_{\left[Q_{m} Q_{m+1}\right]} G^{K}(u-x, v-y, z)((u-x) \mathrm{d} v-(v-y) \mathrm{d} u) \\
& =\sum_{m=0}^{q-1}\left(\overrightarrow{Q_{m} Q_{m+1}} \times \overrightarrow{Q_{m} P}\right) \cdot \overrightarrow{\mathbf{n}} \int_{0}^{1} G^{K}\left(Q_{m}-P+\left(Q_{m+1}-Q_{m}\right) t\right) \mathrm{d} t
\end{aligned}
$$

\section{Power inverse kernels}

For even power inverse kernels, we shall express the convolution function based on a polygon $\mathfrak{Q}=\left[Q_{0}, \ldots, Q_{q-1}\right]$ in terms of the convolution functions based on the line segments $\left[Q_{m} Q_{m+1}\right]$ forming the boundary of the polygons:

$$
\mathcal{C}_{\mathfrak{Q}}^{\mathfrak{p}^{2 i}}(P)=\frac{1}{2(i-1)} \sum_{m=0}^{q-1}\left(\overrightarrow{Q_{m} Q_{m+1}} \times \overrightarrow{Q_{m} P}\right) \cdot \overrightarrow{\mathbf{n}} \sum_{k=1}^{i-1}\left(\frac{1}{|P \bar{P}|^{2}}\right)^{(i-k)} \mathcal{D}_{\left[Q_{m} Q_{m+1}\right]}^{\mathfrak{p}^{2 k}}(P)
$$

where $\bar{P}$ is the projection of $P$ on the plane of the polygon $\mathfrak{Q}$ and $\overrightarrow{\mathbf{n}}$ is a unit normal to this plane.

As mentioned earlier we have $|P \bar{P}|=\left|\overrightarrow{P Q_{0}} \cdot \overrightarrow{\mathbf{n}}\right|=\left|\overrightarrow{P Q_{m}} \cdot \overrightarrow{\mathbf{n}}\right|$. Thus, besides $\overrightarrow{\mathbf{n}}$, the resulting convolution function depends on

- $\left|Q_{m} Q_{m+1}\right|$ and $\left(\overrightarrow{Q_{m} Q_{m+1}} \times \overrightarrow{Q_{m} P}\right) \cdot \overrightarrow{\mathbf{n}}$ for $0 \leq m \leq q$

- $\left|\overrightarrow{P Q_{0}} \cdot \overrightarrow{\mathbf{n}}\right|$ and $\overrightarrow{Q_{m} Q_{m+1}} \cdot \overrightarrow{Q_{m} P},\left|Q_{m} P\right|^{2}$ for $0 \leq m \leq q$

through arithmetic operations, $q$ squareroots and $2 q$ arctan functions.

The functions $\mathcal{C}_{\mathfrak{Q}}^{\mathfrak{p}^{2 i}}$ quite clearly satisfy a reccurrence formula and this recurrence formula holds for odd $i$ as well. We can thus provide a closed form formula for $\mathcal{C}_{\mathfrak{Q}}^{\mathfrak{p}^{i}}$, for all $i \neq 2$.

\subsection{Reduction to contour integral}

We consider the family of power inverse kernels $\mathfrak{p}^{i}: r \mapsto \frac{1}{r^{i}}$ and following Section 5.1 we look for a particular solution of the partial differential equation

$$
u \frac{\partial G^{\mathfrak{p}^{i}}}{\partial u}+v \frac{\partial G^{\mathfrak{p}^{i}}}{\partial v}+2 G^{\mathfrak{p}^{i}}=\frac{1}{\left(u^{2}+v^{2}+z^{2}\right)^{\frac{i}{2}}}
$$


that is differentiable in the domain of integration. As discussed in Section 5.1 we can choose

$$
G^{\mathfrak{p}^{i}}(u, v, z)=\int_{0}^{1} \frac{t \mathrm{~d} t}{\left(\left(u^{2}+v^{2}\right) t^{2}+z^{2}\right)^{\frac{i}{2}}} .
$$

Using [19], and alternatively [11, Section 5], we obtain the following recurrence on $G^{\mathfrak{p}^{i}}$

$$
i z^{2} G^{\mathfrak{p}^{i+2}}(u, v, z)-(i-2) G^{\mathfrak{p}^{i}}(u, v, z)=\left(\frac{1}{u^{2}+v^{2}+z^{2}}\right)^{\frac{i}{2}}
$$

In particular for $i=2$ the above relationships implies:

$$
G^{\mathfrak{p}^{4}}(u, v, z)=\frac{1}{u^{2}+v^{2}+z^{2}}
$$

We can besides find a closed form for $G^{\mathfrak{p}^{1}}$

$$
G^{\mathfrak{p}^{1}}(u, v, z)=\frac{1}{2} \frac{1}{u^{2}+v^{2}}\left(\sqrt{u^{2}+v^{2}+z^{2}}-\sqrt{z^{2}}\right)=\frac{1}{2} \frac{1}{\sqrt{u^{2}+v^{2}+z^{2}}+\sqrt{z^{2}}} .
$$

The recurrence formula above thus shows that the function $G^{\mathfrak{p}^{i}}, i \neq 2$, are well defined and differentiable for $(u, v, z) \neq(0,0,0)$. We can actually give explicit formulae for those. For $z \neq 0$, we have

$$
G^{\mathfrak{p}^{i}}(u, v, z)= \begin{cases}\frac{1}{2} \frac{1}{u^{2}+v^{2}} \ln \left(\frac{u^{2}+v^{2}+z^{2}}{z^{2}}\right) & \text { when } i=2 \\ \frac{1}{2-i} \frac{1}{u^{2}+v^{2}}\left(\left(\frac{1}{u^{2}+v^{2}+z^{2}}\right)^{\frac{i}{2}-1}-\left(\frac{1}{z^{2}}\right)^{\frac{i}{2}-1}\right) & \text { otherwise }\end{cases}
$$

while for $z=0$, but $u^{2}+v^{2} \neq 0$, we have

$$
G^{\mathfrak{p}^{i}}(u, v, 0)= \begin{cases}\frac{1}{2} \frac{1}{u^{2}+v^{2}} \ln \left(u^{2}+v^{2}\right) & \text { when } i=2 \\ \frac{1}{2-i}\left(\frac{1}{u^{2}+v^{2}}\right)^{\frac{i}{2}} & \text { otherwise. }\end{cases}
$$

\subsection{Convolution of Polygons}

Let us resume with the coordinate set up of Section 5 . Let $P$ be the point of $\mathbb{R}^{3}$ with coordinate $(x, y, z)$. The coordinates of $\bar{P}$, the projection of $P$ on the $(O, \overrightarrow{\mathbf{u}}, \overrightarrow{\mathbf{v}})$ plane, are $(x, y, 0)$. Then consider $Q$ to be the point of $(O, \overrightarrow{\mathbf{u}}, \overrightarrow{\mathbf{v}})$ with coordinates $(u, v, 0)$. Then $z^{2}=|P \bar{P}|^{2}$ and $(u-x)^{2}+(v-y)^{2}+z^{2}=|P Q|^{2}$ so that for the function $G^{\mathfrak{p}^{i}}$ introduced in the previous paragraph satisfies:

$$
i|P \bar{P}|^{2} G^{\mathfrak{p}^{i+2}}(u-x, v-y, z)-(i-2) G^{\mathfrak{p}^{i}}(u-x, v-y, z)=\left(\frac{1}{|P Q|^{2}}\right)^{\frac{i}{2}} .
$$

Thus according to Section 5, when $P \neq \bar{P}$

$$
\left.\mathcal{C}_{\mathfrak{Q}}^{\mathfrak{p}^{i+2}}(P)=\frac{i-2}{i|P \bar{P}|^{2}} \mathcal{C}_{\mathfrak{Q}}^{\mathfrak{p}^{i}}(P)+\frac{1}{i|P \bar{P}|^{2}} \sum_{m=0}^{q-1}\left(\overrightarrow{Q_{m} Q_{m+1}} \times \overrightarrow{Q_{m} P}\right) \cdot \overrightarrow{\mathbf{n}} \mathcal{D}_{\left[Q_{m} Q_{m+1}\right]}^{\mathfrak{p}^{i}}(P)\right),
$$


where the function $\mathcal{D}_{[A B]}^{\mathfrak{p}^{i}}$ was introduced in 3.2 as the convolution function for the line segment $[A B]$ up to multiplication by its length.

We read from this recurrence relationship, by susbtituting $i=2$, that

$$
\mathcal{C}_{\mathfrak{Q}}^{\mathfrak{p}^{4}}(P)=\frac{1}{2|P \bar{P}|^{2}} \sum_{m=0}^{q-1}\left(\overrightarrow{Q_{m} Q_{m+1}} \times \overrightarrow{Q_{m} P}\right) \cdot \overrightarrow{\mathbf{n}} \mathcal{D}_{\left[Q_{m} Q_{m+1}\right]}^{\mathfrak{p}^{2}}(P) .
$$

Besides we can compute that $\mathcal{C}_{\mathfrak{Q}}^{\mathfrak{p}^{3}}(P)$. For that we introduce, for $a>0, a c-b^{2}>0$, and $d>c$ the integral $\mathfrak{J}(a, b, c, d)=\int_{0}^{1} \frac{1}{\sqrt{a t^{2}-2 b t+d}\left(a t^{2}-2 b t+c\right)}=\frac{1}{\sqrt{d-c} \sqrt{a c-b^{2}}}\left[\arctan \left(\frac{\sqrt{d-c}}{\sqrt{a c-b^{2}}} \frac{a t-b}{\sqrt{a t^{2}-2 b t+d}}\right)\right]_{0}^{1}$.

Since

$$
G^{\mathfrak{p}^{3}}(u-x, v-y, z)=\frac{1}{|\bar{P} Q|^{2}}\left(\frac{1}{|P \bar{P}|}-\frac{1}{|P Q|}\right)
$$

then

$\mathcal{C}_{\mathfrak{Q}}^{\mathfrak{p}^{3}}(P)=\sum_{m=0}^{q}\left(\overrightarrow{Q_{m} Q_{m+1}} \times \overrightarrow{Q_{m} P}\right) \cdot \overrightarrow{\mathbf{n}}\left(\frac{1}{|P \bar{P}|} \mathcal{D}_{\left[Q_{m} Q_{m+1}\right]}^{\mathfrak{p}^{2}}(\bar{P})-\mathfrak{J}\left(\left|Q_{m} Q_{m+1}\right|^{2}, \overrightarrow{Q_{m} Q_{m+1}} \cdot \overrightarrow{Q_{m} P},\left|Q_{m} \bar{P}\right|^{2},\left|Q_{m} P\right|^{2}\right)\right)$.

We are thus able to give a closed form expression for $\mathcal{C}_{\mathfrak{Q}}^{\mathfrak{p}^{i}}$ for all $i \neq 2$.

\subsection{Case of even powers}

In the above section we provided a recursive way of obtaining the convolution functions based on a polygon for all power inverse kernels. The case of even power inverse kernels has a nice expression in terms of convolution of line segments solely that we can obtain as follows.

We saw that for $i>1$

$$
G^{\mathfrak{p}^{2 i}}(u, v, z)=\frac{1}{2(1-i)} \frac{1}{u^{2}+v^{2}}\left(\left(\frac{1}{u^{2}+v^{2}+z^{2}}\right)^{i-1}-\left(\frac{1}{z^{2}}\right)^{i-1}\right)
$$

and this can be written as

$$
G^{\mathfrak{p}^{2 i}}(u, v, z)=\frac{1}{2(i-1)} \sum_{k=1}^{i-1}\left(\frac{1}{z^{2}}\right)^{i-k}\left(\frac{1}{u^{2}+v^{2}+z^{2}}\right)^{k}
$$

thanks to the simple factorisation rule $\left(\frac{1}{a}\right)^{i-1}-\left(\frac{1}{b}\right)^{i-1}=\left(\frac{1}{a}-\frac{1}{b}\right) \sum_{k=1}^{i-1}\left(\frac{1}{a}\right)^{k-1}\left(\frac{1}{b}\right)^{i-k-1}=(b-a) \sum_{k=1}^{i-1}\left(\frac{1}{a}\right)^{k}\left(\frac{1}{b}\right)^{i-k}$.

For $z \neq 0$, i.e. $P \neq \bar{P}$, we thus have

$$
G^{\mathfrak{p}^{2 i}}(u-x, v-y, z)=\frac{1}{2(1-i)} \sum_{k=1}^{i-1}\left(\frac{1}{|P \bar{P}|^{2}}\right)^{(i-k)}\left(\frac{1}{|P Q|^{2}}\right)^{k}
$$

while when $P=\bar{P}$ we have

$$
G^{\mathfrak{p}^{2 i}}(u-x, v-y, 0)=\frac{1}{2(1-i)}\left(\frac{1}{|P Q|^{2}}\right)^{i}
$$


Thus, according to Section 5 and 3.2 we have

$$
\mathcal{C}_{\mathfrak{Q}}^{\mathfrak{p}^{2 i}}(P)= \begin{cases}\frac{1}{2(1-i)} \sum_{m=0}^{q-1}\left(\overrightarrow{Q_{m} Q_{m+1}} \times \overrightarrow{Q_{m} P}\right) \cdot \overrightarrow{\mathbf{n}} \sum_{k=1}^{i-1}\left(\frac{1}{|P \bar{P}|^{2}}\right)^{(i-k)} \mathcal{D}_{\left[Q_{m} Q_{m+1}\right]}^{\mathfrak{p}^{2 k}}(P) & \text { when } P \neq \bar{P} \\ \frac{1}{2(1-i)}\left(\overrightarrow{Q_{m} Q_{m+1}} \times \overrightarrow{Q_{m} P}\right) \cdot \overrightarrow{\mathbf{n}} \mathcal{D}_{\left[Q_{m} \underline{p}_{m+1}{ }^{2 i}\right.}(P) & \text { when } P=\bar{P} \notin \mathfrak{Q} .\end{cases}
$$

We have thus expressed convolution functions based on the polygon $\mathfrak{Q}$ in terms of convolution functions based on the line segments forming the boundary of the polygons.

\subsection{Illustration}

For the kernel $\mathfrak{p}^{4}: r \mapsto \frac{1}{r^{4}}$ the convolution function for a polygon $\mathfrak{Q}=\left[Q_{0}, \ldots, Q_{q-1}\right]$ is the simplest:

$$
\mathcal{C}_{\mathfrak{Q}}^{\mathfrak{p}^{4}}(P)=\frac{1}{2} \sum_{m=0}^{q-1} \frac{1}{|P \bar{P}|^{2}}\left(\overrightarrow{Q_{m} P} \wedge \overline{Q_{m} Q_{m+1}}\right) \cdot \overrightarrow{\mathbf{n}} \mathcal{D}_{\left[Q_{m} Q_{m+1}\right]}^{\mathfrak{p}^{2}}(P)
$$

and recall that

$$
\mathcal{D}_{[A B]}^{\mathfrak{p}^{2}}(P)=\frac{1}{\sqrt{a c-b^{2}}}\left(\arctan \left(\frac{a-b}{\sqrt{a c-b^{2}}}\right)+\arctan \left(\frac{b}{\sqrt{a c-b^{2}}}\right)\right) .
$$

where

$$
a=|A B|^{2}, \quad b=\overrightarrow{A B} \cdot \overrightarrow{A P}, c=|A P|^{2} .
$$

The resulting convolution surface is shown for a regular pentagon and a non convex polygon in Figure 6 and 7 .
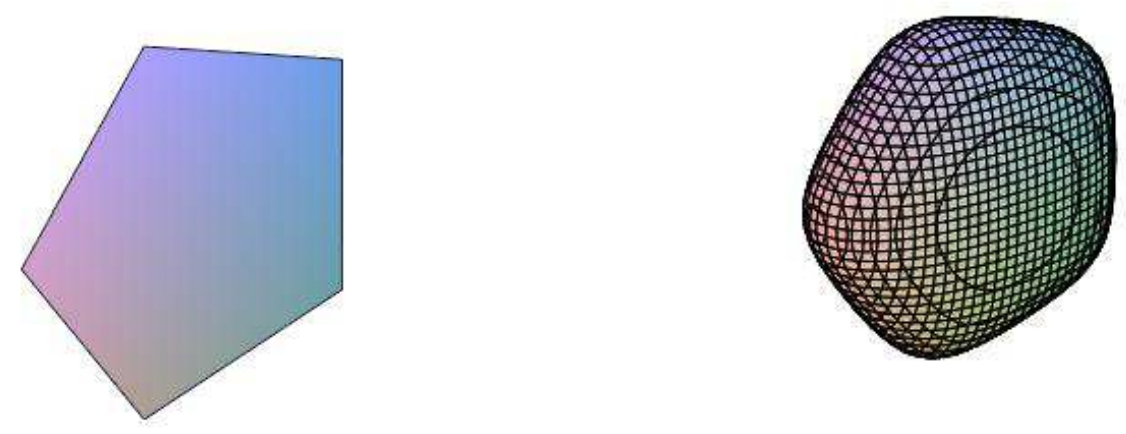

Figure 6: Pentagon and convolution surface generated with the $\mathfrak{p}^{4}$ kernel. 

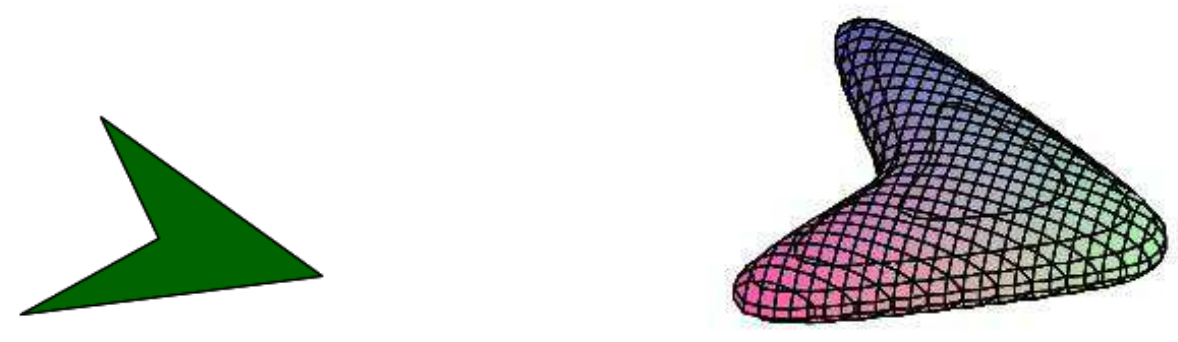

Figure 7: Non-convex polygon and convolution surface generated with the $\mathfrak{p}^{4}$ kernel.

\section{Cauchy kernels}

The formulae and recurrence for the convolution functions based on the polygon $\mathfrak{Q}=\left[Q_{0}, \ldots, Q_{q-1}\right]$ with Cauchy kernels can be obtained just as above. In particular, for the subfamily of kernels $\mathfrak{c}_{s}^{2 i}$ with $i>1$ we have:

$$
\mathcal{C}_{\mathfrak{Q}}^{\mathfrak{c}_{s}^{2 i}}(P)=\frac{1}{2(i-1)} \sum_{m=0}^{q-1}\left(\overrightarrow{Q_{m} Q_{m+1}} \times \overrightarrow{Q_{m} P}\right) \cdot \overrightarrow{\mathbf{n}} \sum_{k=1}^{i-1}\left(\frac{1}{1+s|P \bar{P}|^{2}}\right)^{(i-k)} \mathcal{D}_{\left[Q_{m} Q_{m+1}\right]}^{c_{s}^{2 k}}(P)
$$

where $\bar{P}$ is the projection of $P$ on the plane of the polygon $\mathfrak{Q}$ and $\overrightarrow{\mathbf{n}}$ is a unit normal to this plane. So in particular, for the original Cauchy kernel [15], we have

$$
\mathcal{C}_{\mathfrak{Q}}^{\mathfrak{c}_{s}^{4}}(P)=\frac{1}{2\left(1+s|P \bar{P}|^{2}\right)} \sum_{m=0}^{q-1}\left(\overrightarrow{Q_{m} Q_{m+1}} \times \overrightarrow{Q_{m} P}\right) \cdot \overrightarrow{\mathbf{n}} \mathcal{D}_{\left[Q_{m} Q_{m+1}\right]}^{\mathfrak{c}_{s}^{2}}(P) .
$$

This actually explains and generalizes the remark made by Sherstyuk [21, Section 2.4.4] about triangles: the convolution function is a sum of three step-functions, one for each edge of the triangle. This is illustrated for the equilateral triangle $\mathfrak{Q}=[(2,0,0),(-1, \sqrt{3}, 0),(-1,-\sqrt{3}, 0)]$ in Figure 8 where one of the so-called step function is drawn.

\section{Compact support kernels}

We apply the strategy described in Section 5 to the case of compact kernels. The gain here is even bigger than for infinite support kernels. For infinite support kernels we had avoided the triangulation, that actually leads to redundant computation, as was illustrated in Section 7. Here we gain by avoiding the computation of the geometry of the intersection of a sphere with the polygon (triangle) for the evaluation of the convolution function at a given point. We only need to compute the intersection of a sphere with line segments, which is well worked out. 

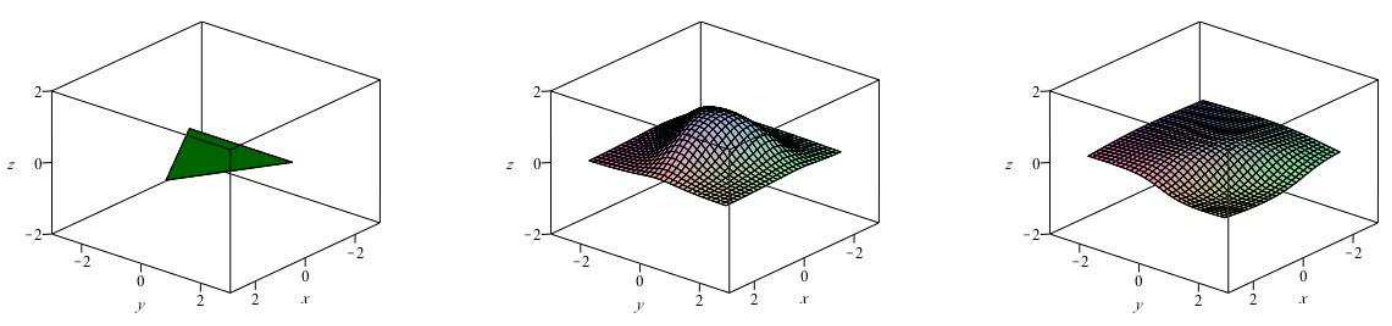

Figure 8: An equilateral triangle (left), the graph of the convolution function above it (middle) and the step-function like contribution of a side of the triangle to it (right).

As in Section 6 we can obtain a recurrence formulae for the whole family of compact support kernels we introduced. We nonetheless feel that the family of even power, that are piecewise polynomial kernels, is suficient for practical purposes. Those are furthermore easier to establish.

\subsection{Reduction to contour integral}

We consider the family of kernels

$$
\mathfrak{k}_{R}^{i}: r \mapsto \begin{cases}\left(1-\frac{r^{2}}{R^{2}}\right)^{\frac{i}{2}} & \text { if } r<R \\ 0 & \text { otherwise }\end{cases}
$$

for $i \geq 3$. Following Section 5.1 we look for a particular solution of the partial differential equation

$$
u \frac{\partial G_{R}^{\mathfrak{k}_{R}^{i}}}{\partial u}+v \frac{\partial G^{\mathfrak{k}_{R}^{i}}}{\partial v}+2 G^{\mathfrak{k}_{R}^{i}}= \begin{cases}\left(1-\frac{u^{2}+v^{2}+z^{2}}{R^{2}}\right)^{\frac{i}{2}} & \text { for } u^{2}+v^{2}+z^{2} \leq R^{2} \\ 0 & \text { for } u^{2}+v^{2}+z^{2} \geq R^{2}\end{cases}
$$

that is continuously differentiable. Applying the homotopy operator of Section 5.1 we obtain:

$$
G^{\mathfrak{k}_{R}^{i}}(u, v, z)= \begin{cases}\frac{1}{(2+i)} \frac{R^{2}}{u^{2}+v^{2}}\left(\left(1-\frac{z^{2}}{R^{2}}\right)^{\frac{i}{2}+1}-\left(1-\frac{u^{2}+v^{2}+z^{2}}{R^{2}}\right)^{\frac{i}{2}+1}\right) & \text { when } u^{2}+v^{2}+z^{2} \leq R^{2} \\ \frac{R^{2}}{(2+i)} \frac{\left(1-\frac{z^{2}}{R^{2}}\right)^{\frac{i}{2}+1}}{u^{2}+v^{2}} & \text { when } u^{2}+v^{2} \geq R^{2}-z^{2}>0 \\ 0 & \text { when } z^{2} \geq R^{2}\end{cases}
$$

For even $i$ and thanks to the simple factorisation rule $a^{i+1}-b^{i+1}=(a-b) \sum_{k=0}^{i} a^{i-k} b^{k}$, the latter can be 
written

$$
G^{\mathfrak{k}_{R}^{2 i}}(u, v, z)= \begin{cases}\frac{1}{2(1+i)} \sum_{k=0}^{i}\left(1-\frac{z^{2}}{R^{2}}\right)^{i-k}\left(1-\frac{u^{2}+v^{2}+z^{2}}{R^{2}}\right)^{k} & \text { when } u^{2}+v^{2}+z^{2} \leq R^{2} \\ \frac{1}{2(1+i)}\left(1-\frac{z^{2}}{R^{2}}\right)^{i+1} \frac{R^{2}}{u^{2}+v^{2}} & \text { when } u^{2}+v^{2} \geq R^{2}-z^{2}>0 \\ 0 & \text { when } z^{2} \geq R^{2}\end{cases}
$$

from which it is seen to be well defined and continuously differentiable on $\mathbb{R}^{3}$.

\subsection{Convolution of Polygons}

Let us resume with the coordinate set up of Section 5 . Let $P$ be the point of $\mathbb{R}^{3}$ with coordinate $(x, y, z)$. The coordinates of $\bar{P}$, the projection of $P$ on the $(O, \overrightarrow{\mathbf{u}}, \overrightarrow{\mathbf{v}})$ plane, are $(x, y, 0)$. Then consider $Q$ be the point of $(O, \overrightarrow{\mathbf{u}}, \overrightarrow{\mathbf{v}})$ with coordinates $(u, v, 0)$. The previous function can be written in terms of geometric quantities involving those points:

$$
G^{\mathfrak{k}_{R}^{2 i}}(u-x, v-y, z)= \begin{cases}\frac{1}{2(1+i)} \sum_{k=0}^{i}\left(1-\frac{|P \bar{P}|^{2}}{R^{2}}\right)^{i-k}\left(1-\frac{|P Q|^{2}}{R^{2}}\right)^{k} & \text { when }|P Q| \leq R \\ \frac{1}{2(1+i)}\left(1-\frac{|P \bar{P}|^{2}}{R^{2}}\right)^{i+1} \frac{R^{2}}{|\bar{P} Q|^{2}} & \text { when }|\bar{P} Q|^{2} \geq R^{2}-|P \bar{P}|^{2}>0 \\ 0 & \text { when }|P \bar{P}| \geq R\end{cases}
$$

If the distance of $P$ to the plane where the polygon sits is bigger than $R$ then the convolution function is zero. Otherwise, assuming $|P \bar{P}|<R$, we have:

$$
\begin{aligned}
\mathcal{C}_{\mathfrak{Q}}^{\mathfrak{k}_{R}^{2 i}}(P) & =\frac{1}{2(1+i)} \sum_{m=0}^{q-1}\left(\overrightarrow{Q_{m}^{P} Q_{m+1}^{P}} \times \overrightarrow{Q_{m}^{P} P}\right) \cdot \overrightarrow{\mathbf{n}} \sum_{k=0}^{i}\left(1-\frac{|P \bar{P}|^{2}}{R^{2}}\right)^{i-k} \mathcal{D}_{\left[Q_{m}^{P} Q_{m+1}^{P}\right.}^{\mathfrak{k}^{2 k}}(P) \\
& +\frac{R^{2}}{2(1+i)}\left(1-\frac{|P \bar{P}|^{2}}{R^{2}}\right)^{i+1} \sum_{m=0}^{q-1}\left(\overrightarrow{Q_{m} Q_{m}^{P}} \times \overrightarrow{Q_{m} P}\right) \cdot \overrightarrow{\mathbf{n}} \mathcal{D}_{\left[Q_{m} Q_{m}^{2}\right]}^{\mathfrak{p}^{2}}(\bar{P}) \\
& +\frac{R^{2}}{2(1+i)}\left(1-\frac{|P \bar{P}|^{2}}{R^{2}}\right) \sum_{m=0}^{i+1}\left(\overrightarrow{Q_{m+1}^{P} Q_{m+1}} \times \overrightarrow{Q_{m+1}^{P} P}\right) \cdot \overrightarrow{\mathbf{n}} \mathcal{D}_{\left[Q_{m+1}^{\mathfrak{p}^{2}}\right.}^{q-1} Q_{m+1}^{P}(\bar{P})
\end{aligned}
$$

where, for each $m, Q_{m}^{P}$ and $Q_{m+1}^{P}$ are the end points of the intersection of the sphere of radius $R$ centered at $P$ with the line segment $\left[Q_{m} Q_{m+1}\right]$. When $Q_{m}^{P}=Q_{m}$ or $Q_{m+1}=Q_{m+1}^{P}$ the second or third term is zero.

We illustrate the convolution function obtained with $\mathfrak{k}_{R}^{i}$ by plotting the convolution surface around two polygons which are at a distance 2 and where $R$ is taken to be 1 in Figure 9 . The generated convolution surface are continuously differentiable but flat towards the center of the face. This is to be compared with the convolution surface obtained with a power inverse kernel.

\section{Conclusion and perspective}

We have provided closed form formulae for the convolution functions based on any planar polygons and for whole families of compact or infinite support kernels. Our approach avoids the prior triangulation of the 

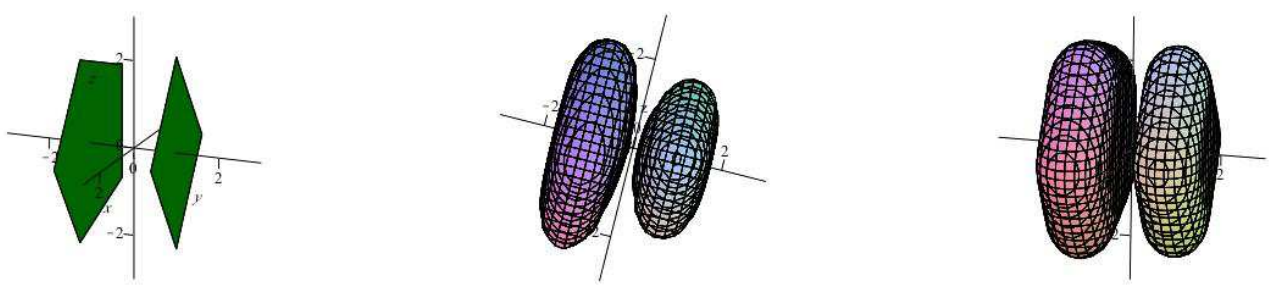

Figure 9: Convolution surfaces based on parallel polygons (left) with a cubic inverse kernel (middle) and compact support kernel (right) where $R$ is chosen to be less than the distance bewteen the two polygons. An inward bulge appear in between the polygons for the cubic inverse kernel, while the convolution surface is flat towards the center of the polygons on both sides for the compact support kernel.

polygons and the recursive formulation allows to sharpen or smooth the shape around the skeleton iteratively. Our strategy avoids the complicated geometric computations previously needed for compact support kernels.

We can extend the ideas of this paper to introduce some geometry or weight functions on the polygons. This would allow for full generality of amenable shapes. The difficulty relies then on having a continuous weight function accross the skeleton, i.e. along the edges shared by more than one polygon. This works quite naturally if we consider only linear weights over triangles and is the subject of a future article. Higher degree weight functions with more complicated polygons appear as rather more subtle task.

As Green's theorem generalizes to Stokes theorem we envision to use non planar skeleton patches, such as spherical cups.

\section{References}

[1] M. Berger and B. Gostiaux. Differential geometry: manifolds, curves, and surfaces, volume 115 of Graduate Texts in Mathematics. Springer-Verlag, New York, 1988. Translated from the French by Silvio Levy.

[2] A. Bernhardt, A. Pihuit, M.-P. Cani, and L. Barthe. Matisse: Painting 2D regions for modeling freeform shapes. In EUROGRAPHICS Workshop on Sketch-Based Interfaces and Modeling, pages 57-64, 2008.

[3] J. Blinn. A generalization of algebraic surface drawing. ACM Transactions on Graphics, 1(3):235-256, 1982.

[4] J. Bloomenthal and K. Shoemake. Convolution surfaces. Computer Graphics, 25(4):251-255, 1991.

[5] Manuel Bronstein. Symbolic integration. I, volume 1 of Algorithms and Computation in Mathematics. Springer-Verlag, Berlin, second edition, 2005.

[6] J. Chun-Kin Fong C.-L. Tai, H. Zhang. Prototype modeling from sketched silhouettes based on convolution surfaces. Computer Graphics Forum, 23(1):71-84, 2004.

[7] M-P. Cani and S. Hornus. Subdivision curve primitives: a new solution for interactive implicit modeling. In International Conference on Shape Modeling and Applications, pages 82-88. IEEE Computer Society Press, 2001. 
[8] C. Gasquet and P. Witomski. Fourier analysis and applications, volume 30 of Texts in Applied Mathematics. Springer-Verlag, New York, 1999.

[9] K. O. Geddes, S. R. Czapor, and G. Labahn. Algorithms for computer algebra. Kluwer Academic Publishers, Boston, MA, 1992.

[10] S. Hornus, A. Angelidis, and M.-P. Cani. Implicit modelling using subdivision curves. Visual Comput., 19(2-3):94-104, 2003.

[11] E. Hubert and M.-P. Cani. Convolution surfaces based on polygonal curve skeletons. Journal of Symbolic Computation, 2011. http://hal.inria.fr/inria-00429358/en/.

[12] X. Jin and C.-L. Tai. Analytical methods for polynomial weighted convolution surfaces with various kernels. Computers and Graphics, 26:437-447, 2002.

[13] X. Jin, C.-L. Tai, and H. Zhang. Implicit modeling from polygon soup using convolution. The Visual Computer, 25(3):279-288, 2009.

[14] Tao Ju. Fixing geometric errors on polygonal models: A survey. J. Comput. Sci. Technol., 24(1):19-29, 2009.

[15] J. McCormack and A. Sherstyuk. Creating and rendering convolution surfaces. The International Journal of the Eurographics Association, Computer Graphics Forum, 17(2):113-120, 1998.

[16] J. Milnor. Morse theory. Based on lecture notes by M. Spivak and R. Wells. Annals of Mathematics Studies, No. 51. Princeton University Press, Princeton, N.J., 1963.

[17] H. K. Nickerson, D. C. Spencer, and N. E. Steenrod. Advanced calculus. D. Van Nostrand Co., Inc., Toronto-Princeton, N.J.-New York-London, 1959.

[18] H. Nishimura, M. Hirai, T. Kawai, T. Kawata, I. Shirakawa, and K. Omura. Objects modeling by distribution function and a method of image generation (in japanese). The Transactions of the Institute of Electronics and Communication Engineers of Japan, J68-D(4):718-725, 1985.

[19] A. P. Prudnikov, Yu. A. Brychkov, and O. I. Marichev. Integrals and series. Vol. 1. Gordon \& Breach Science Publishers, New York, 1986.

[20] C. Shen, J. F. O'Brien, and J. R. Shewchuk. Interpolating and approximating implicit surfaces from polygon soup. ACM Trans. Graph., 23(3):896-904, 2004.

[21] A. Sherstyuk. Convolution Surfaces in Computer Graphics. PhD thesis, Monash University, Australia, 1999.

[22] A. Sherstyuk. Interactive shape design with convolution surfaces. In Shape Modeling International '99, pages 56-65, 1999.

[23] A. Sherstyuk. Kernel functions in convolution surfaces: a comparative analysis. The Visual Computer, 15(4), 1999.

[24] K. Siddiqi and S. M. Pizer, editors. Medial Representations: Mathematics, Algorithms and Applications. Number 37 in Computational Imaging. Springer, 2008.

[25] I. M. Singer and J. A. Thorpe. Lecture notes on elementary topology and geometry. Scott, Foresman and Co., Glenview, Ill., 1967.

[26] B. Wyvill and K. van Overveld. Tiling techniques for implicit skeletal models. SIGGRAPH Course Notes, 11:1-26., 1996.

[27] G. Wyvill, C. McPheeters, and B. Wyvill. Data structure for soft objects. The Visual Computer, 2(4):227-234, 1986. 